\title{
Two-stage genome-wide association study identifies a novel susceptibility locus associated with melanoma
}

\author{
Katherine J. Ransohoff ${ }^{1, *}$, Wenting Wu ${ }^{2,}{ }^{*}$, Hyunje G. Cho ${ }^{1}$, Harvind C. Chahal ${ }^{1}$, Yuan \\ Lin², Hong-Ji Dai'2,3, Christopher I. Amos"4, Jeffrey E. Lee ${ }^{5}$, Jean Y. Tang ${ }^{1}$, David A. \\ Hinds $^{6}$, Jiali Han ${ }^{2,7, \#}$, Qingyi Wei ${ }^{8, \#}$, Kavita Y. Sarin ${ }^{1, \#}$ \\ ${ }^{1}$ Department of Dermatology, Stanford University School of Medicine, Stanford, CA, USA \\ ${ }^{2}$ Department of Epidemiology, Richard M. Fairbanks School of Public Health, Melvin and Bren Simon Cancer Center, Indiana \\ University, Indianapolis, IN, USA \\ ${ }^{3}$ Department of Epidemiology and Biostatistics, Tianjin Medical University Cancer Hospital and Institute, National Clinical \\ Research Center for Cancer, Tianjin and Key Laboratory of Cancer Prevention and Therapy, Tianjin, China \\ ${ }^{4}$ Department of Community and Family Medicine, Geisel School of Medicine, Dartmouth College, Hanover, NH, USA \\ ${ }^{5}$ Department of Surgical Oncology, The University of Texas M.D. Anderson Cancer Center, Houston, TX, USA \\ ${ }^{6}$ 23andMe Inc., Mountain View, CA, USA \\ ${ }^{7}$ Department of Epidemiology, Harvard T.H. Chan School of Public Health, Boston, MA, USA \\ ${ }^{8}$ Duke Cancer Institute, Department of Medicine, Duke University, Durham, NC, USA \\ *Co-first author \\ "Co-senior author
}

Correspondence to: Kavita Y. Sarin, email: ksarin@stanford.edu

Keywords: melanoma, genome-wide association study, single nucleotide polymorphism, susceptibility loci, BASP1

Received: December 02, 2016

Accepted: January 27, 2017

Published: February 09, 2017

\section{ABSTRACT}

Genome-wide association studies have identified 21 susceptibility loci associated with melanoma. These loci implicate genes affecting pigmentation, nevus count, telomere maintenance, and DNA repair in melanoma risk. Here, we report the results of a two-stage genome-wide association study of melanoma. The stage 1 discovery phase consisted of 4,842 self-reported melanoma cases and 286,565 controls of European ancestry from the 23andMe research cohort and the stage 2 replication phase consisted of 1,804 melanoma cases and 1,026 controls from the University of Texas M.D. Anderson Cancer Center. We performed a combined metaanalysis totaling 6,628 melanoma cases and 287,591 controls. Our study replicates 20 of 21 previously known melanoma-loci and confirms the association of the telomerase reverse transcriptase, TERT, with melanoma susceptibility at genomewide significance. In addition, we uncover a novel polymorphism, rs187843643 (OR $\left.=1.96 ; 95 \% \mathrm{CI}=[1.54,2.48] ; P=3.53 \times 10^{-8}\right)$, associated with melanoma. The SNP rs187842643 lies within a noncoding RNA $177 \mathrm{~kb}$ downstream of BASP1 (brain associated protein-1). We find that BASP1 expression is suppressed in melanoma as compared with benign nevi, providing additional evidence for a putative role in melanoma pathogenesis.

\section{INTRODUCTION}

Melanoma represents $1 \%$ of cutaneous malignancies, affecting approximately 76,000 people per year in the U.S, but causes the majority of skin cancer deaths [1]. Environmental risk factors such as ultraviolet radiation and fair skin pigmentation contribute to melanoma development [2]. About 10-12\% of melanomas occur in familial clusters; to date, approximately half of the genes responsible for these clusters have been 
identified [3]. Prior genome-wide association studies (GWAS) have identified 21 loci associated with cutaneous melanoma (Supplementary Table 2). These previous studies implicate nevus count, pigmentation, telomere homeostasis, tumor suppression and DNA repair in melanoma development.

Crowd-sourced data has recently been utilized to identify susceptibility loci for a wide range of disease phenotypes [4]. Here, we utilize crowd-sourced data in a two-stage genome-wide association meta-analysis for melanoma, totaling 6,628 cases and 287,591 controls. In this GWAS, we replicate 20 of 21 previously identified melanoma-associated loci and discover one novel susceptibility locus at genome-wide significance.

\section{RESULTS}

Stage 1 consisted of 4,842 self-reported melanoma cases and 286,565 controls of European ancestry from the 23 andMe, Inc. research cohort (Table 1). A validation of self-report of melanoma history using the same 23 andMe survey questions with adjudicated medical records revealed a sensitivity of $100 \%$ and specificity of 98.8\% ( $p<0.0001$; Fisher's exact test) (Supplementary Table 1). The most significant melanoma-associated SNP at each locus $\left(P<10^{-5}\right)$ was identified, resulting in nine index single nucleotide polymorphisms (SNPs) (Table 2, Figure 1). Stage 2 consisted of 1,804 melanoma cases and 1,026 non-hispanic controls from the MD Anderson Cancer Center. Four of the nine index SNPS were replicated in the stage 2 analysis $(P<0.05)$. Although some loci did not reach statistical significance in stage 2 , their 95\% confidence intervals (for odds ratios) overlapped with the corresponding stage 1 confidence intervals.

A combined meta-analysis of stage 1 and stage 2, totaling 6,628 melanoma cases and 287,591 controls identified nine susceptibility loci reaching genomewide significance (Table 2; $P<5 \times 10^{-8}$ ). Out of 21 previously known melanoma-associated loci, 20 were replicated at nominal $p$ value $(P<0.05$, logistic regression) (Supplementary Table 2 ). We identified one novel melanoma-susceptibility locus, located at 5p15.1 (rs187843643; BASP1-[]) $\left(\mathrm{P}=3.53 \times 10^{-8}\right.$; OR = 1.96, logistic regression). Further information on methods and imputation quality control can be found in Supplementary Methods, and Supplementary Tables 3-4. QQ plots, forest plots, and regional association plots are provided in Supplementary Figures 1-3.

To measure the heritability of melanoma attributable to the genome-wide significant SNPs from stage 1, we calculated the familial relative risk for melanoma outlined by the Cancer Oncological Gene Environment Study (COGS) [5]. The nine loci explained 9\% of familial melanoma risk. The novel SNP rs187843643 contributes to $1 \%$ of familial melanoma risk.

\section{DISCUSSION}

Of the nine genome-wide significant SNPs resulting from the combined meta-analysis, eight were at previously identified loci associated with melanoma: $M C 1 R$, SLC45A2, RALY, MTAP, IRF4-EXOC2, TERT, TYR, and $M X 2$. These loci are associated with pigmentation phenotype and nevus count. The pigmentation loci include 5p13.2 (SLC45A2), 11q14.3 (TYR), 16q24.3 (MC1R) and 20q11.22 (RALY/ASIP); nevi-associated loci are 9p21.3 (CDKN2A-MTAP), 22q13.1 (PLA2G6). The SNP rs6059655, intergenic near $R A L Y-A S I P$, is associated with facial pigmentation spots [6] and rs35407 (SLC45A2), is associated with pigmentation and melanoma risk $[7,8]$. It is important to note that some loci associated with pigmentation phenotype may also contribute to melanoma risk independent of pigmentation. For example, in addition to affecting hair color, previous studies have demonstrated that primary human melanocytes with $M C 1 R$ variants have impaired DNA-repair [9].

Our study identified one novel SNP not previously associated with melanoma, rs187843643. While rs 187843643 did not reach statistical significance in stage 2 , likely due to low allele frequency and limited number of stage 2 cases, its $95 \%$ confidence interval (for odds ratios) overlapped with corresponding stage 1 confidence intervals. Rs187843643, located at 5p15.1, lies $177 \mathrm{~kb}$ downstream of brain abundant membrane attached signal protein $1\left(B A S P 1 ; P=3.53 \times 10^{-08} ;\right.$ OR $=1.96$, logistic regression) and within a poorly characterized long noncoding RNA, RP11-321E2.4. BASP1 is a proteincoding gene, with several PEST motifs, which are associated with proteins with high turnover. The role of BASP1 protein in cancer has not been well established. One study demonstrated an association between increased BASP1 expression in stage III and stage IV melanoma tumor cells and improved melanoma survival [10]. Consistent with a protective role for BASP1 in melanoma, we found that BASP1 expression was suppressed in melanoma $(N=45)$ as compared with benign nevi $(N=$ 18 ) by 0.26 fold ( $P=0.007$, moderated t-statistic) using publicly available expression data (GEO, GDS1375/ GSE3189) (Figure 2) [11, 12]. BASP1 expression has also been shown to be downregulated in hepatocellular carcinoma via epigenetic regulation [13]. This implicates a potential tumor-suppressive role for BASP1 in melanoma. Interestingly, this locus was not identified by the recent meta-analysis by Law et al., potentially due to variability in the imputation panels and QC filters [14].

Telomere homeostasis has been previously associated with melanoma risk. Multiple studies now support the association between longer telomere length and increased melanoma susceptibility, as well with increased nevus count $[15,16]$. In addition, telomererelated loci have been associated with risk of melanoma in GWAS including: ATM, TERT [17], and more recently, 
Table 1: Gender and age of melanoma cases and controls from GWAS

\begin{tabular}{|c|c|c|c|c|c|c|c|}
\hline & Status & $n(\%)$ & Male (\%) & Age $\leq 30$ yr & Age 30-45 & Age 45-60 & Age $>60$ \\
\hline \multirow{2}{*}{$\begin{array}{l}\text { 23andMe (Stage } \\
(n=291,389)\end{array}$} & Cases & $4,824(1.66)$ & $2587(53.6)$ & $99(2)$ & $476(9.9)$ & 1,203 (24.9) & $3,056(63.3)$ \\
\hline & Controls & $286,565(98.3)$ & $154,517(53.9)$ & 40,028 (13.9) & $8,4188(29.3)$ & $7,8037(27.2)$ & $8,4312(29.4)$ \\
\hline \multirow{2}{*}{$\begin{array}{l}\text { MD Anderson (Stage 2) } \\
(n=\mathbf{2 , 8 3 0})\end{array}$} & Cases & $1,804(63.7)$ & $1,060(58.8)$ & $142(7.9)$ & $441(24.5)$ & 684 (37.9) & $536(29.7)$ \\
\hline & Controls & $1,026(36.3)$ & $613(59.8)$ & $69(6.7)$ & $231(22.5)$ & $486(47.4)$ & $240(23.4)$ \\
\hline \multirow{2}{*}{$\begin{array}{l}\text { Combined analysis } \\
(n=294,219)\end{array}$} & Cases & $6,628(2.25)$ & $3677(2.3)$ & $241(3.6)$ & $9,171(13.8)$ & $1,887(28.4)$ & $3,592(54.2)$ \\
\hline & Controls & $287,591(97.7)$ & $155,130(97.7)$ & 40,097 (13.9) & $84,419(29.3)$ & $78,523(27.3)$ & $84,552(29.4)$ \\
\hline
\end{tabular}

Counts and percentages for cases and controls $(n(\%))$ are listed above, stratified by stage of GWAS. We also report number and percentage of male subjects, subjects with age $<30$ years, subjects with age 30-45 years, subjects with age 45-60 years, and subjects with age $>60$ years.

Table 2: Loci reaching genome-wide significance in melanoma GWAS

\begin{tabular}{|c|c|c|c|c|c|c|c|c|c|c|c|}
\hline \multirow[b]{2}{*}{ SNP } & \multirow[b]{2}{*}{ Region } & \multirow[b]{2}{*}{ Gene } & \multirow[b]{2}{*}{ Maj/Min } & \multirow{2}{*}{$\begin{array}{c}\text { Avg. } \\
\text { imputation } \\
\mathbf{r}^{2}\end{array}$} & \multirow[b]{2}{*}{ MAF $^{1}$} & \multicolumn{2}{|c|}{ 23\&Me (Stage 1) } & \multicolumn{2}{|c|}{ MD Anderson (Stage 2) } & \multicolumn{2}{|c|}{ Meta-Analysis ${ }^{2}$} \\
\hline & & & & & & $P$ & $\begin{array}{c}\text { OR } \\
{\left[\mathrm{CI}^{3}\right]}\end{array}$ & $P$ & $\begin{array}{c}\text { OR } \\
{\left[\mathbf{C I}^{3}\right]}\end{array}$ & $P$ & $\begin{array}{c}\text { OR } \\
{\left[\mathrm{CI}^{3}\right]}\end{array}$ \\
\hline rs1805007 & $16 \mathrm{q} 24.3$ & $M C 1 R$ & $\mathrm{C} / \mathrm{T}$ & 1.00 & 0.07 & $3.8 \times 10^{-28}$ & $\begin{array}{c}1.47 \\
{[1.38,1.57]}\end{array}$ & $4.88 \times 10^{-8}$ & $\begin{array}{c}1.72 \\
{[1.41,2.09,]}\end{array}$ & $4.24 \times 10^{-37}$ & $\begin{array}{c}1.50 \\
{[1.41,1.59]}\end{array}$ \\
\hline rs 35407 & $5 \mathrm{p} 13.2$ & $S L C 45 A 2$ & $\mathrm{G} / \mathrm{A}$ & 0.98 & 0.03 & $5.6 \times 10^{-22}$ & $\begin{array}{c}0.46 \\
{[0.39,0.55]}\end{array}$ & $6.44 \times 10^{-3}$ & $\begin{array}{c}0.57 \\
{[0.38,0.85]}\end{array}$ & $3.42 \times 10^{-19}$ & $\begin{array}{c}0.48 \\
{[0.41,0.56]}\end{array}$ \\
\hline rs6059655 & $20 \mathrm{q} 11.22$ & $R A L Y-A S I P$ & $\mathrm{G} / \mathrm{A}$ & 0.99 & 0.07 & $3.1 \times 10^{-18}$ & $\begin{array}{c}1.37 \\
{[1.28,1.46]}\end{array}$ & $1.02 \times 10^{-4}$ & $\begin{array}{c}1.44 \\
{[1.20,1.74]}\end{array}$ & $5.04 \times 10^{-23}$ & $\begin{array}{c}1.38 \\
{[1.29,1.47]}\end{array}$ \\
\hline rs201131773 & $9 \mathrm{p} 21.3$ & MTAP & $\mathrm{I} / \mathrm{D}$ & 0.99 & 0.48 & $2.4 \times 10^{-17}$ & $\begin{array}{c}1.19 \\
{[1.15,1.24]}\end{array}$ & - & - & $2.5 \times 10^{-17}$ & $\begin{array}{c}1.19 \\
{[1.15,1.24]}\end{array}$ \\
\hline rs62389423 & $6 \mathrm{p} 25.3$ & $\begin{array}{c}\text { IRF4-[]-- } \\
\text { EXOC2 }\end{array}$ & $\mathrm{G} / \mathrm{A}$ & 0.78 & 0.14 & $9.7 \times 10^{-13}$ & $\begin{array}{c}1.26 \\
{[1.18,1.34]}\end{array}$ & $7.77 \times 10^{-2}$ & $\begin{array}{c}1.15 \\
{[0.98,1.34]}\end{array}$ & $9.14 \times 10^{-14}$ & $\begin{array}{c}1.24 \\
{[1.17,1.31]}\end{array}$ \\
\hline rs139996880 & $5 \mathrm{p} 15.33$ & $T E R T$ & $\mathrm{G} / \mathrm{A}$ & 0.65 & 0.16 & $2.4 \times 10^{-11}$ & $\begin{array}{c}1.25 \\
{[1.18,1.34]}\end{array}$ & $0.48^{*}$ & $\begin{array}{c}1.06^{*} \\
{[0.90,1.26]}\end{array}$ & $7.16 \times 10^{-12}$ & $\begin{array}{c}1.26 \\
{[1.18,1.34]}\end{array}$ \\
\hline rs 1393350 & $11 \mathrm{q} 14.3$ & $T Y R$ & $\mathrm{G} / \mathrm{A}$ & 1.00 & 0.27 & $2.7 \times 10^{-11}$ & $\begin{array}{c}1.17 \\
{[1.11,1.22]}\end{array}$ & $8.47 \times 10^{-3}$ & $\begin{array}{c}1.18 \\
{[1.04,1.33]}\end{array}$ & $3.65 \times 10^{-13}$ & $\begin{array}{c}1.17 \\
{[1.12,1.22]}\end{array}$ \\
\hline rs 45430 & $21 \mathrm{q} 22.3$ & $M X 2$ & $\mathrm{~T} / \mathrm{C}$ & 1.00 & 0.34 & $1.1 \times 10^{-7}$ & $\begin{array}{c}0.89 \\
{[0.85,0.93]}\end{array}$ & 0.11 & $\begin{array}{c}0.91 \\
{[0.82,1.02]}\end{array}$ & $2.89 \times 10^{-8}$ & $\begin{array}{c}0.89 \\
{[0.86,0.93]}\end{array}$ \\
\hline rs 187843643 & $5 \mathrm{p} 15.1$ & $\begin{array}{c}B A S P 1--- \\
{[]^{* *}}\end{array}$ & $\mathrm{C} / \mathrm{T}$ & 0.74 & 0.01 & $6.0 \times 10^{-7}$ & $\begin{array}{c}1.96 \\
{[1.54,2.50]}\end{array}$ & 0.52 & $\begin{array}{c}1.69 \\
{[0.34,8.38]}\end{array}$ & $3.53 \times 10^{-8}$ & $\begin{array}{c}1.96 \\
{[1.54,2.48]}\end{array}$ \\
\hline
\end{tabular}

SNPs that met genome-wide significance $\left(P<5 \times 10^{-8}\right)$ in the overall meta-analysis are listed. Additionally, we report genetic locus, nearest genes, major allele, minor allele, minor allele frequency (MAF) in stage 1 controls, average imputation $r^{2}$ (a measure of imputation quality) for stage 1 , and odds ratio (OR) with $P$ value for each stage, calculated with respect to the minor allele. Stage 1 included 4,842 melanoma cases and 286,565 controls from 23andMe. Stage 2 included 1,804 melanoma cases and 1,026 controls from the MD Anderson Cancer Center. The combined fixed-effect meta-analysis, totaled 6,628 melanoma cases and 287,591 controls. Statistics for effect heterogeneity $\left(P_{\text {het }}\right.$ and $\left.I^{2}\right)$ are included in Supplementary Table 4. All subjects were from the US and of European ancestry.

${ }^{1} \mathrm{MAF}=$ minor allele frequency in stage 1 controls

${ }^{2}$ Meta-analysis $=$ Combined $23 \& \mathrm{Me}+\mathrm{MD}$ Anderson

${ }^{3} \mathrm{CI}=95 \%$ confidence interval

$* *=$ Not previously associated with melanoma risk

$*=$ Imputation $\mathrm{r}^{2}=0.2968$ in MD Anderson Dataset.

$O B F C 1$ [14]. TERT, the catalytic subunit of telomerase, plays a critical role in maintaining telomere length and has been shown to support cancer progression through both telomere-dependent and telomere-independent mechanisms [18]. Polymorphisms at the TERT locus have been associated with melanoma in multiple candidate studies and rare mutations in TERT have been identified in high-incident melanoma families [3]. Our GWAS found an association between the TERT marker rs139996880 (5p15.33) and increased melanoma risk at genome-wide 
significance $\left(P=7.16 \times 10^{-12} ; \mathrm{OR}=1.26\right)$ confirming the association of TERT with melanoma. Our findings further support the importance of telomere homeostasis in melanoma.

This two-stage GWAS validates the use of consumer self-report data as a platform for discovery of new cancer- related genes, provides confirmation of 20 out of 21 of the previously known melanoma-associated loci, and identifies one novel susceptibility locus $(5 \mathrm{p} 15.1 ; B A S P 1)$ which confers a 1.96-fold increase in risk of melanoma. Further exploration into the role of the BASP1 locus in melanoma pathogenesis is warranted.

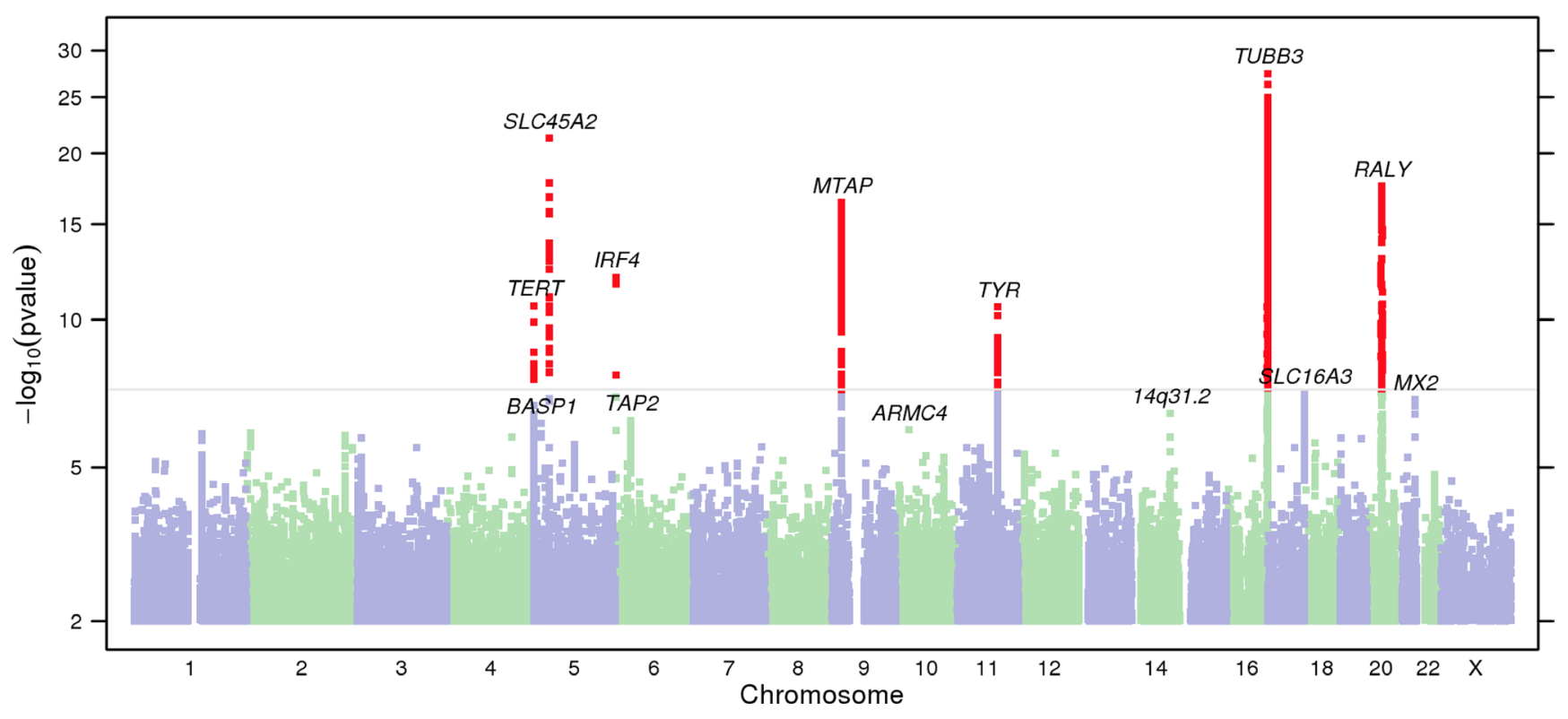

Figure 1: Manhattan plot of stage 1 GWAS analysis of melanoma from 23andMe dataset. Total stage 1 GWAS analysis included 4,842 melanoma cases and 286,565 controls. SNPs with $P<5 \times 10^{-8}$ are shown in red. Loci with smallest $P<10^{-6}$ are labeled with the name of the nearest gene. In stage 1, 13 loci reached genome-wide significance after adjusting for genomic control. One novel locus, 5p15.1 (BASP1-[]) was genome-wide significant in the overall meta-analysis (Table 1).

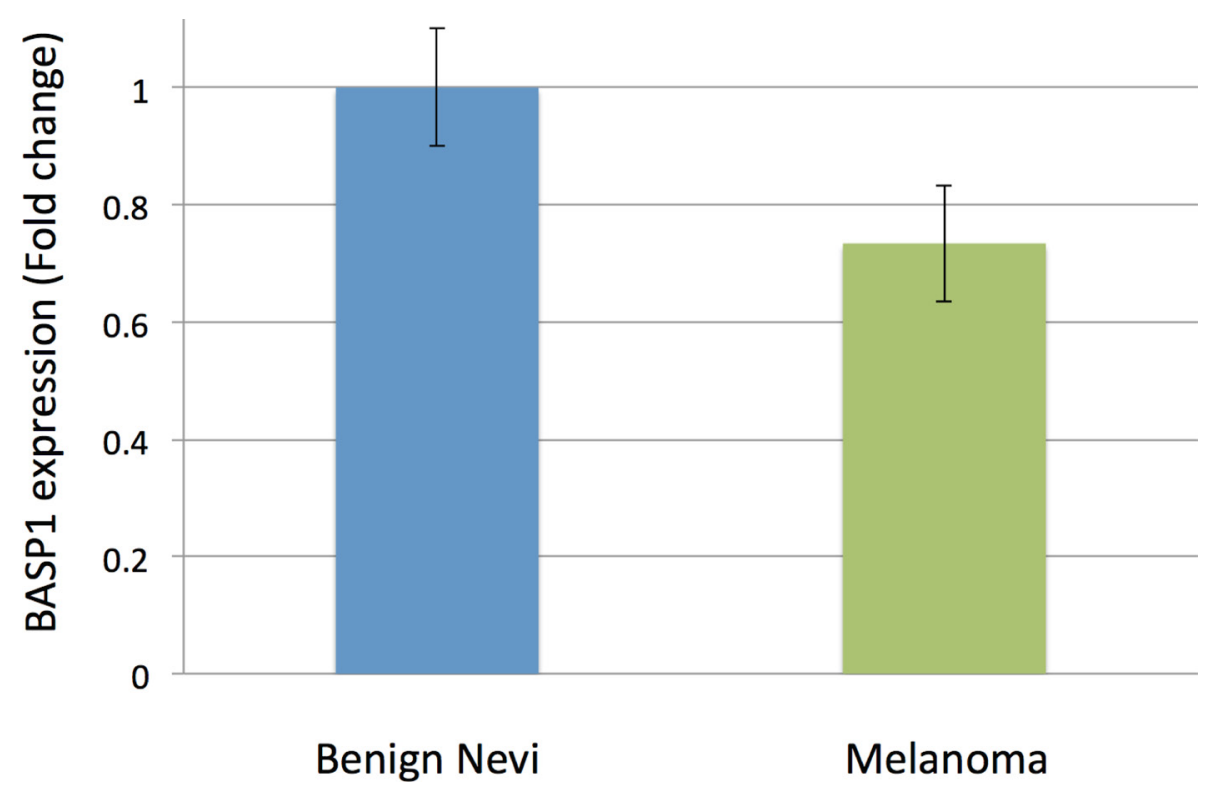

Figure 2: Fold-change in BASP1 expression in benign nevi $(n=18)$ vs. melanoma samples $(n=45)$ using publicly available data in GEO (GDS1375). Using benign nevi as a reference, BASP1 is suppressed by about 0.26 fold in melanoma samples $(P=0.007$, moderated t-statistic). Error bars represent standard error. Data from GEO, GDS1375. 


\section{MATERIALS AND METHODS}

\section{Stage 1 study design and population}

23andMe, Inc. (Mountain View, CA), a personal genetics company, provided free access to aggregated genetic and phenotypic information for stage 1 of the GWAS. 23andMe research participants provided informed consent, in accordance with 23 andMe's human subjects protocol (reviewed and approved by Ethical and Independent Review Services, a AAHRPP-accredited IRB). 23andMe gathers genetic information for research by genotyping sample material provided by customers who have consented to research; phenotypic information is collected via online surveys taken by research participants. Inclusion and exclusion criteria are discussed below.

\section{Stage 1 genome-wide association analysis}

Association analysis for stage 1 was performed using logistic regression, assuming an additive model for allelic effects. The analysis was adjusted for age, sex, and population stratification (using the first five principal components), generating the following model:

1) Melanoma diagnosis $\sim$ age + sex + pc. $0+$ pc. $1+$ pc. $2+p c .3+$ pc. $4+$ genotype. Analyses were restricted to individuals with $>97 \%$ European ancestry from the local ancestry analysis to address outlier. Five principal components were extensively evaluated to verify robustness and its use in capturing ancestry structure within Europe. The association test $p$ value was computed using a likelihood ratio test. Results for the $\mathrm{X}$ chromosome were computed similarly, with male genotypes coded as if they were homozygous diploid for the observed allele. Additional to principal component analysis, test statistics were adjusted for genomic control to correct for residual population stratification persisting after principal component analysis; the genomic control inflation factor was 1.016 (computed from the median $p$ value for results that passed quality control). Regions of interest were defined by identifying SNPs with $P<10^{-5}$, then grouping these into intervals separated by gaps of at least $250 \mathrm{~kb}$, and choosing the SNP with the smallest $p$ value within each interval.

\section{Sensitivity and specificity of stage 1 self-reported data}

To assess the validity of self-reported phenotypic data in stage 1,23andMe surveys (pertaining to skin cancer history and pigmentation) were randomly administered to 188 patients seen in Stanford outpatient clinics. The survey answers were then compared to medical records to assess for accuracy with respect to melanoma diagnosis to determine the sensitivity and specificity of the survey responses. $P$ values were determined using a Fisher's exact test due to the presence of low frequency events. This sub-study was approved by the Stanford
University Institutional Review Board with a waiver of documentation of informed consent.

\section{Stage 2 study design and population}

The study participants were from a hospital-based case-control study of melanoma, for which cases were recruited from among non-Hispanic white patients and controls at MD Anderson between March 1998 and August 2008. Samples and data were available from 931 melanoma patients and 1,026 cancer-free controls (friends of other patients reporting to clinics), which were frequency-matched on age and sex, had completed a comprehensive skin lifestyle questionnaire, and had passed quality control filters for genotyping. This questionnaire was administered by an interviewer to $70 \%$ of patients and controls and was self-administered for the remaining $30 \%$. An additional case series comprising 873 individuals presenting for treatment for melanoma at MD Anderson was also included, bringing the total number of melanoma patients to 1,804 . The study protocols were approved by the Institutional Review Board at MD Anderson and informed consent was obtained from all participants.

\section{Stage 2 genome-wide association analysis}

Association analysis with risk of melanoma of genotyped SNPs or most likely genotypes from the imputation study was performed using the PLINK logistic and -covar options. A logistic regression model was built to measure the additive effect of each SNP on susceptibility to melanoma. A likelihood ratio test was performed under the null hypothesis of $\mathrm{x} 2$ distribution with one degree of freedom. The first two PCs were included to adjust for population structure [19].

\section{Meta-analysis}

For each SNP, associations in stage 1 and stage 2 were combined in an inverse-variance-weighted metaanalysis using the METAL software [20]. Imputation qualities across batches in 23 andMe chips were tested for to pick up variants that have differences in behavior across arrays. Heterogeneity of per-SNP effect sizes in studies contributing to the stage 1 , stage 2 , and the overall meta-analysis was assessed. All $\mathrm{R}^{2}$ and $\mathrm{D}^{\prime}$ values between individual SNPs were calculated based on the 1000 Genomes Pilot 1 dataset, CEU Population (http:// www.broadinstitute.org/mpg/snap/ldsearchpw.phpL.

\section{Proportion of familial relative risk}

We have used the formula for calculating the proportion of FRR as outlined by the Cancer Oncological Gene-environment Study (COGS) [5]. The odds ratios derived from our meta-analysis of stage 1 and stage 2 are assumed to be relative risks. We estimated the proportion 
of the familial relative risk (FRR) explained by each SNP $\left(\mathrm{FRR}_{\text {snp }}\right)$ as:

$$
\text { 1) } \mathrm{FRR}_{\text {snp }}=\left(p r^{2}+q\right) /(p r+q)^{2}
$$

Here, the risk allele and alternative allele frequencies are $p$ and $q$, respectively, and $r$ is the odds ratio for the risk allele. Allele frequencies are derived from the stage 1 population data. Assuming that the loci combine multiplicatively and are not in linkage disequilibrium, the combined effect of all loci is given by:

$$
\text { 2) } \lambda_{\mathrm{T}}=\prod_{k} \lambda_{\mathrm{K}}
$$

Here, the product is across all loci. The proportion of the familial relative risk attributable to the SNPs, on a $\log$ scale, is then given by $\log \left(\lambda_{T}\right) / \log \left(\lambda_{P}\right)$, where $\lambda_{P}$ is the familial relative risk observed in epidemiological studies, assuming an $\lambda_{P}$ for melanoma of 2.19 [14].

\section{Gene expression analysis}

Processed gene expression data for melanoma and nevi (GSE3189) was obtained from the Gene Expression Omnibus (GEO, http://www.ncbi.nlm.nih.gov/geo/). Forty-five melanoma samples and 18 controls (18 benign nevi) were included [12]. Each gene of interest was selected by its proximity to one of the novel risk alleles. For each dataset, Geo2R, which employs a linear-based model for microarray analysis, was utilized to compare gene expression between melanoma and normal skin controls [21]. Significant results were defined as instances of differential gene expression (in melanoma tissue relative to control) reaching $P<0.05$ the dataset.

\section{Abbreviations}

SNP, single nucleotide polymorphism; GWAS, genome-wide association study.

\section{ACKNOWLEDGMENTS}

We would like to thank the research participants and employees of 23 andMe for making this work possible. The authors assume full responsibility for analyses and interpretation of these data.

\section{CONFLICTS OF INTEREST}

David Hinds is an employee of 23 andMe, Inc.

\section{FUNDING}

This work was supported by the National Human Genome Research Institute of the National Institutes of Health (grant number R44HG006981), and in part by NIH R01 CA49449, P01 CA87969, UM1 CA186107, UM1 CA167552, the Stanford TRAM (Translational Research and Applied Medicine) Program and the Dermatology Foundation Career Development Award (KS).

\section{Authors' contributions}

Conception and design of study: J Han, DA Hinds, JY Tang, KY Sarin.

Acquisition of data: DA Hinds, J Han, Q Wei, KY Sarin, CI Amos, JE Lee.

Analysis and/or interpretation of data: KJ Ransohoff, W Wu, HG Cho, H Chahal, Y Lin, HJ Dai, DA Hinds, J Han, Q Wei, KY Sarin.

Drafting the manuscript: KJ Ransohoff, HG Cho, J Han, KY Sarin.

Revising manuscript critically for important intellectual content: KJ Ransohoff, HG Cho, W Wu, DA Hinds, J Han, KY Sarin.

\section{REFERENCES}

1. Nikolaou V, Stratigos AJ. Emerging trends in the epidemiology of melanoma. Br J Dermatol. 2014; 170:11-19.

2. Berwick M, Buller DB, Cust A, Gallagher R, Lee TK, Meyskens F, Pandey S, Thomas NE, Veierod MB, Ward S. Melanoma Epidemiology and Prevention. Cancer Treat Res. 2016; 167:17-49.

3. Ransohoff KJ, Jaju PD, Tang JY, Carbone M, Leachman S, Sarin KY. Familial skin cancer syndromes: Increased melanoma risk. J am Acad Dermutol. 2016; 74:423-434.

4. Do CB, Tung JY, Dorfman E, Kiefer AK, Drabant EM, Francke U, Mountain JL, Goldman SM, Tanner CM, Langston JW, Wojcicki A, Eriksson N. Web-based genomewide association study identifies two novel loci and a substantial genetic component for Parkinson's disease. PLoS genetics. 2011; 7:e1002141.

5. Bahcall O. Common variation and heritability estimates for breast, ovarian and prostate cancers. Nature Genetics. 2013. doi: 10.1038/ngicogs.1.

6. Jacobs LC, Hamer MA, Gunn DA, Deelen J, Lall JS, van Heemst D, Uh HW, Hofman A, Uitterlinden AG, Griffiths CE, Beekman M, Slagboom PE, Kayser M, et al. A Genome-Wide Association Study Identifies the Skin Color Genes IRF4, MC1R, ASIP, and BNC2 Influencing Facial Pigmented Spots. J Invest Dermatol. 2015; 135:1735-1742.

7. Fernandez LP, Milne RL, Pita G, Aviles JA, Lazaro P, Benitez J, Ribas G. SLC45A2: a novel malignant melanomaassociated gene. Hum Mutat. 2008; 29:1161-1167.

8. Duffy DL, Zhao ZZ, Sturm RA, Hayward NK, Martin NG, Montgomery GW. Multiple pigmentation gene polymorphisms account for a substantial proportion of risk of cutaneous malignant melanoma. J Invest Dermatol. 2010; 130:520-528.

9. Kadekaro AL, Leachman S, Kavanagh RJ, Swope V, Cassidy P, Supp D, Sartor M, Schwemberger S, Babcock G, 
Wakamatsu K, Ito S, Koshoffer A, Boissy RE, et al. Melanocortin 1 receptor genotype: an important determinant of the damage response of melanocytes to ultraviolet radiation. FASEB journal. 2010; 24:3850-3860.

10. Kaehler KC, Politz O, Henderson D, Ulbrich HF, Hauschild A, Mund C, Egberts F. Novel DNA methylation markers with potential prognostic relevance in advanced malignant melanoma identified using COBRA assays. Melanoma Res. 2015; 25:225-231.

11. Talantov D, Mazumder A, Yu JX, Briggs T, Jiang Y, Backus J, Atkins D, Wang Y. Novel genes associated with malignant melanoma but not benign melanocytic lesions. Clin Cancer Res. 2005; 11:7234-7242.

12. Edgar R, Domrachev M, Lash AE. Gene Expression Omnibus: NCBI gene expression and hybridization array data repository. Nucleic Acids Res. 2002; 30:207-210.

13. Tsunedomi R, Ogawa Y, lizuka N, Sakamoto K, Tamesa T, Moribe T, Oka M. The assessment of methylated BASP1 and SRD5A2 levels in the detection of early hepatocellular carcinoma. Int J Oncol. 2010; 36:205-212.

14. Law MH, Bishop DT, Lee JE, Brossard M, Martin NG, Moses EK, Song F, Barrett JH, Kumar R, Easton DF, Pharoah PDP, Swerdlow AJ, Kypreou KP, et al. Genomewide meta-analysis identifies five new susceptibility loci for cutaneous malignant melanoma. Nature Genet. 2015; 47:987-995.

15. Nan H, Qureshi AA, Prescott J, De Vivo I, Han J. Genetic variants in telomere-maintaining genes and skin cancer risk. Hum Gen. 2011; 129:247-253.
16. Burke LS, Hyland PL, Pfeiffer RM, Prescott J, Wheeler W, Mirabello L, Savage SA, Burdette L, Yeager M, Chanock S, De Vivo I, Tucker MA, Goldstein AM, et al. Telomere length and the risk of cutaneous malignant melanoma in melanoma-prone families with and without CDKN2A mutations. PloS one. 2013; 8:e71121.

17. Barrett JH, Iles MM, Harland M, Taylor JC, Aitken JF, Andresen PA, Akslen LA, Armstrong BK, Avril MF, Azizi E, Bakker B, Bergman W, Bianchi-Scarra G, et al. Genome-wide association study identifies three new melanoma susceptibility loci. Nat Genet. 2011; 43:11081113.

18. Artandi SE, DePinho RA. Telomeres and telomerase in cancer. Carcinogenesis. 2010; 31:9-18.

19. Amos CI, Wang LE, Lee JE, Gershenwald JE, Chen WV, Fang S, Kosoy R, Zhang M, Qureshi AA, Vattathil S, Schacherer CW, Gardner JM, Wang Y, et al. Genomewide association study identifies novel loci predisposing to cutaneous melanoma. Hum Mol Gen. 2011; 20:5012-5023.

20. Willer CJ, Li Y, Abecasis GR. METAL: fast and efficient meta-analysis of genomewide association scans. Bioinformatics (Oxford, England). 2010; 26:2190-2191.

21. Davis S, Meltzer PS. GEOquery: a bridge between the Gene Expression Omnibus (GEO) and BioConductor. Bioinformatics (Oxford, England). 2007; 23:1846-1847. 